\title{
Interpreting Aerodynamics of a Transonic Impeller from Static Pressure Measurements
}

\author{
Fangyuan Lou (D), John Charles Fabian, and Nicole Leanne Key $\mathbb{C}$ \\ Purdue University, West Lafayette, IN 47907, USA \\ Correspondence should be addressed to Fangyuan Lou; louf@purdue.edu
}

Received 22 November 2017; Accepted 27 February 2018; Published 10 April 2018

Academic Editor: Jingyin Li

Copyright (C) 2018 Fangyuan Lou et al. This is an open access article distributed under the Creative Commons Attribution License, which permits unrestricted use, distribution, and reproduction in any medium, provided the original work is properly cited.

This paper investigates the aerodynamics of a transonic impeller using static pressure measurements. The impeller is a highspeed, high-pressure-ratio wheel used in small gas turbine engines. The experiment was conducted on the single stage centrifugal compressor facility in the compressor research laboratory at Purdue University. Data were acquired from choke to near-surge at four different corrected speeds (Nc) from $80 \%$ to $100 \%$ design speed, which covers both subsonic and supersonic inlet conditions. Details of the impeller flow field are discussed using data acquired from both steady and time-resolved static pressure measurements along the impeller shroud. The flow field is compared at different loading conditions, from subsonic to supersonic inlet conditions. The impeller performance was strongly dependent on the inducer, where the majority of relative diffusion occurs. The inducer diffuses flow more efficiently for inlet tip relative Mach numbers close to unity, and the performance diminishes at other Mach numbers. Shock waves emerging upstream of the impeller leading edge were observed from $90 \%$ to $100 \%$ corrected speed, and they move towards the impeller trailing edge as the inlet tip relative Mach number increases. There is no shock wave present in the inducer at $80 \%$ corrected speed. However, a high-loss region near the inducer throat was observed at $80 \%$ corrected speed resulting in a lower impeller efficiency at subsonic inlet conditions.

\section{Introduction}

High-pressure-ratio centrifugal compressors have been widely used in turbochargers and turboshaft engines because of their compact size, high efficiency, and wide operating range. Modern turbochargers and turboshaft engines are continuously pushing the boundary of pressure ratio and flow capacity. Size limitations on the outer diameter lead to larger rotational speeds and result in transonic flow conditions at the compressor inlet. Compared to the impeller flow with subsonic inlet conditions, the flow in transonic impellers is more complex due to the presence of shock waves. It is believed that, in a transonic centrifugal compressor, additional losses could be generated due to the interaction between the shock waves and blade surface boundary layer/tip clearance flow. Investigations by Rodgers and Sapiro [1] showed a drop in impeller peak efficiency (PE) with the increase of impeller inlet shroud relative Mach number, and this has prompted several investigations on transonic impellers.
The flow inside a centrifugal impeller with transonic inlet conditions was studied by Senoo et al. [2] with static pressure measurements along the impeller shroud and later by Hayami et al. [3] with velocity measurements inside the inducer using a laser-2-focus velocimeter (L2FV). At subsonic flow conditions, the impeller stalled at low flow rates, and flow separation was observed. However, the flow separation was limited to the semi-open area between the inducer leading edge (LE) and the throat. Thus, it allowed for a stable operation range without deterioration of impeller efficiency. At supersonic inlet conditions, the results revealed two shock waves inside the inducer: one detached shock wave in front of the main blade LE and the other on the pressure surface in the flow passage. Additionally, the highest polytropic efficiency was observed at conditions near impeller stall, indicating that shock waves do not severely deteriorate impeller performance.

Krain et al. $[4,5]$ conducted detailed measurements of the flow field inside a transonic backswept impeller first using L2FV and later using 3-component laser Doppler velocimetry 
(LDV). The relative inducer tip Mach number was 1.3. Results showed that the first flow instability occurred near the shroud in the inducer due to the interference of shock waves with the tip leakage vortex. The tip leakage flow extended over the full passage as the flow moved towards the impeller exit, and the flow pattern at the impeller exit seemed to be dominated by the wake formed by the tip leakage flow.

Ibaraki et al. [6-8] and Marconcini et al. [9, 10] studied the flow field inside a transonic turbocharger impeller at both design and off-design conditions. The impeller features a double-splitter design. The relative Mach number at the impeller leading edge tip was about 1.3, the same as the case studied by Krain et al. [4, 5], and the results showed similar flow patterns as well. The low-velocity regions were first observed near the shroud/suction-side area in the inducer due to the interaction between the shock wave and the tip leakage flow. This interaction enhances the total pressure loss in the inducer. At the exit of impeller, the low-velocity regions formed by the tip leakage vortex dominated the flow field.

Higashimori et al. [11] carried out detailed flow measurements on an impeller with a relative tip Mach number of 1.6 at the inducer, and a CFD simulation was also conducted for comparison. The research was mainly focused on the inducer flow, and measurements at four traverse planes were acquired. Results showed that an oblique shock was formed at the leading edge of the inducer as the flow entered the impeller, and a passage shock appeared at the inducer throat. Reversed flow near the shroud in the inducer was present because of the interaction between the shock wave and the tip leakage flow. Buffaz and Trébinjac [12] investigated the flow in the inducer of a transonic centrifugal compressor from both velocity measurements using LDV and time-resolved static pressure measurements along the impeller shroud at the inducer section. It was concluded that the change in flow pattern from choke to surge is mainly due to the change in the tip leakage flow.

In summary, flow in transonic impellers is more complex due to the presence of shock waves; reversed flow near the shroud in the inducer may occur due to the presence of shock waves; the tip leakage flow plays a more important role both within the impeller and at the exit of impeller; flow instabilities may start further upstream at the throat of inducer due to the interaction of shock waves and tip leakage flow.

\section{Scope of the Paper}

There are conflicting results on transonic impellers due to the limited impeller geometries on which the previous research was performed. For example, results from Senoo et al. [2] showed that the effect of the interaction between the shock wave and tip leakage flow is confined within the semi-open area of the impeller near the LE. However, results from Krain et al. $[4,5]$ and Ibaraki et al. [6-8] showed an extended effect of the interaction between shock wave and tip leakage flow at the impeller exit.

This paper presents detailed analysis of transonic impeller aerodynamics using data acquired from both steady and time-resolved static pressure measurements along the impeller shroud. The impeller is a high-speed, highpressure-ratio wheel used in aerogas turbine engines. The results help in understanding the aerodynamics in transonic impellers and also enrich the database of research in impeller aerodynamics in the open literature.

\section{Facility and Instrumentation}

The present study was performed on the single stage centrifugal compressor (SSCC) facility with an APU-style inlet in the compressor research laboratory at Purdue University. The facility includes a 1400 horsepower AC electric motor, a 30.46 : 1 ratio gearbox, an exhaust plenum, and a Honeywell experimental compressor. Details of the SSCC facility were documented by Lou et al. [13]. Figure 1 shows the crosssection of the compressor and the location of performance instrumentation. The entire stage includes an inlet housing, an unshrouded impeller, a vaned diffuser, a bend, and deswirl. The impeller features 17 main blades and 17 splitter blades. The compressor performance is calculated based on the total pressure and total temperature measurements at compressor inlet and deswirl exit, as indicated in Figure 1. The design operating speed for the compressor is about 45,000 rpm, and the total pressure ratio for the entire stage is on the order of 6.5 .

Figure 2 shows the pressure instrumentation along the impeller shroud. Static pressure taps were equally spaced along the impeller shroud from the LE to trailing edge (TE) at 10 meridional locations. Additionally, 10 fast-response pressure transducers were installed along the impeller chord from impeller LE to $40 \%$ meridional position.

The static pressures were measured using Scanivalve pressure modules of high accuracy, and a high-speed data acquisition system was used for acquiring the signals from the fast-response transducers. The analog millivolt signals from the fast-response pressure transducers are amplified using Precision Filter Cards 28118. The amplified signals are then digitized using National Instruments cards of model 6358. Since the blade passing frequency is on the order of $25 \mathrm{KHz}$ at design speed, a sampling rate of $1 \mathrm{MHz}$ was used for digitizing the unsteady pressure measurements. This gives more than 1300 data points per rotor revolution, and, thus, it provides very good spatial resolution in the time domain that enables detailed flow features in the impeller to be captured.

Experimental data were acquired from choke to nearsurge at four different corrected speeds (Nc) from $80 \%$ to $100 \%$ design speed. A constant 5.1\% exducer clearance in the axial direction relative to impeller tip blade height was maintained throughout the test. The impeller-diffuser match bleed flow was closed, and a constant $1 \%$ back face bleed flow relative to the compressor inlet flow rate was maintained throughout the test.

\section{Results and Discussions}

Compressor steady performance results and a detailed analysis of impeller performance using steady and time-resolved static pressure measurements are presented. The steady 


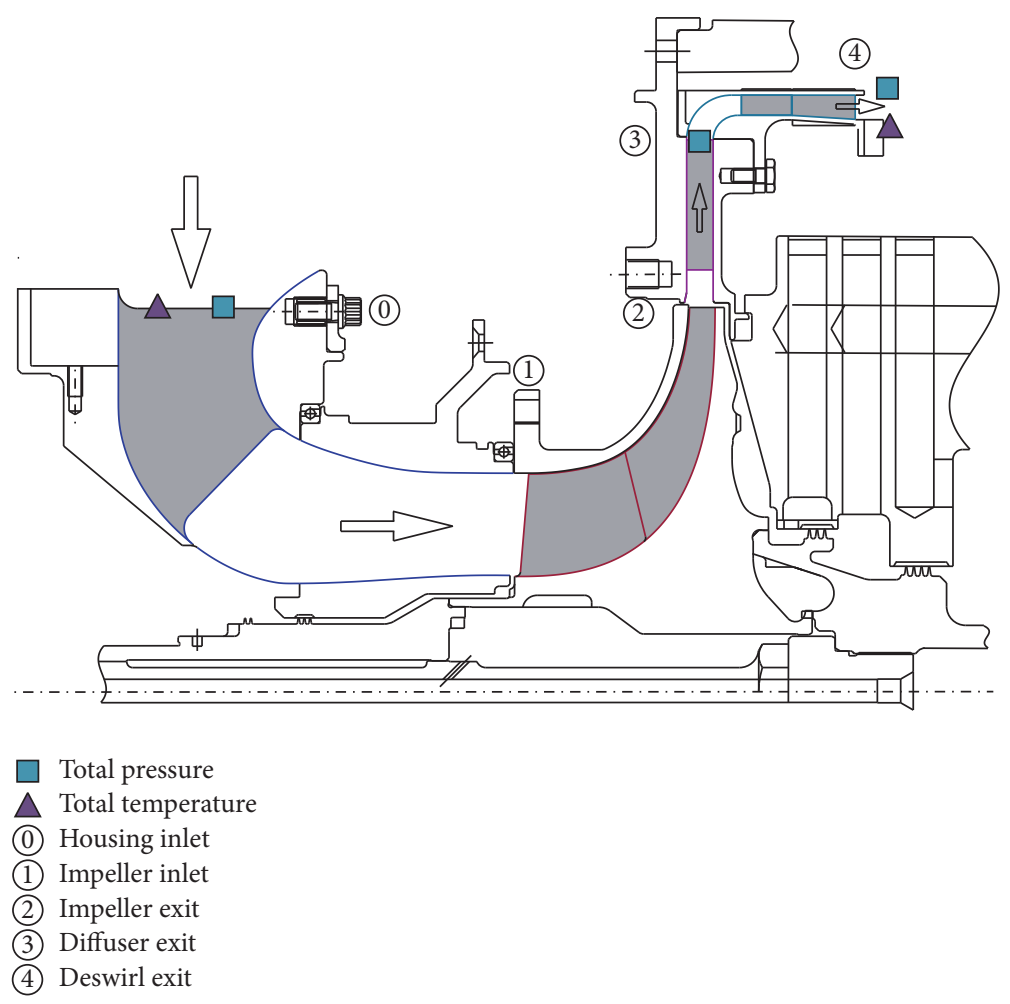

FIGURE 1: Cross-section of compressor and performance instrumentation.
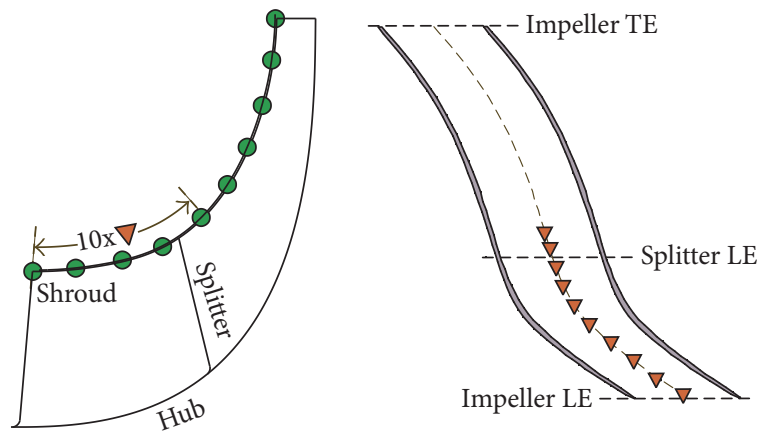

Static pressure tap

Kulite transducer

FIGURE 2: Pressure instrumentation along impeller shroud.

performance is characterized by the total pressure ratio, total temperature ratio, and efficiency. The performance of the entire compressor stage is calculated from the area-averaged inlet and exit conditions measured at stations 0 and 4 . The impeller performance is evaluated using the area-averaged inlet condition measured at station 0 and the impeller exit condition at station 2 , where static pressure measurements are available. The total temperature at the impeller exit is assumed to be the same as that measured at the deswirl exit (station 4) based on the adiabatic assumption. The impeller exit total pressure is derived from the measured total temperature at the deswirl exit, the inlet mass flow rate, and the areaaveraged static pressure measured at the impeller trailing edge using the continuity and the turbomachinery Euler equation [14]. The compressor corrected conditions (speed and mass flow rate) [15] and efficiency [16] are calculated using properties for humid air retrieved from REPROP [17]. Results presented in this section are normalized using the operating condition at design point. The compressor inlet pressure is measured using highly accurate 2.5 psid modules with an uncertainty less than $0.12 \%$. Compressor exit pressure is measured using the 100 psid modules with uncertainty less than $0.05 \%$ of full scale. This renders an uncertainty in the total pressure ratio less than $0.2 \%$ from $80 \%$ to $100 \%$ corrected speed. The mass flow rate is measured using a calibrated bell mouth with an uncertainty less than $0.5 \%$.

Figure 3 shows the normalized total pressure versus the normalized corrected mass flow rate. Results for both the impeller and the entire stage from $80 \%$ to $100 \%$ corrected speed are presented. The peak efficiency (PE) conditions are represented by the solid green symbols. The low loading (LL) conditions are represented by the solid blue symbols. The choke conditions are shown as solid red symbols. Those color schemes are consistent in Figures 4-8. Regardless of the variation in the total pressure ratio for the entire compressor stage relative to the changes in the loading conditions, the total pressure ratio for the impeller stays very constant along the choke line due to the choked flow in the diffuser.

Figure 4 shows the performance of the impeller and entire compressor stage in terms of isentropic efficiency. Compared to the entire stage, the impeller operates more efficiently over the entire operating range from choke to near-surge. There is no obvious deterioration in impeller efficiency along the 


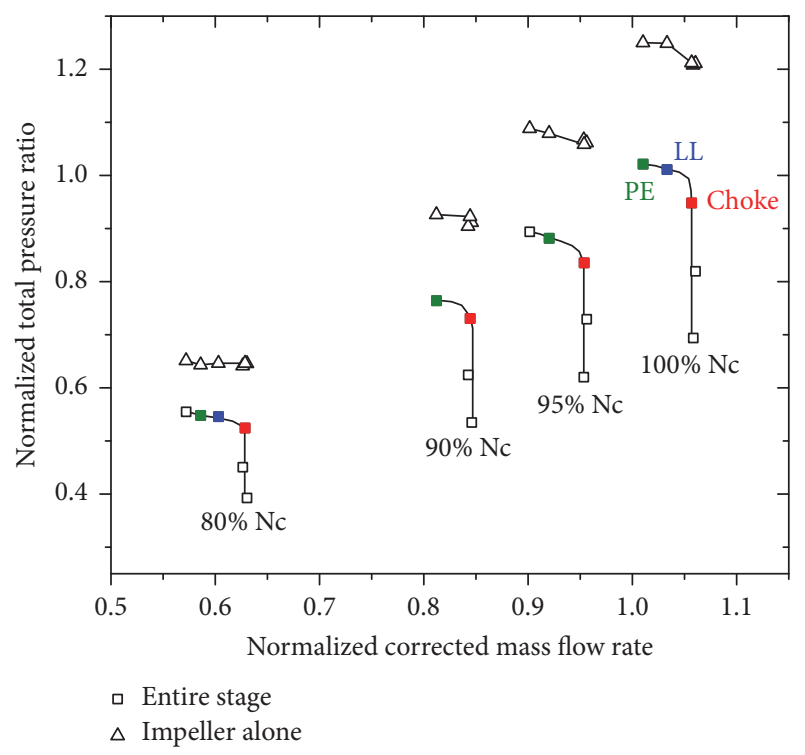

FIgURE 3: Total pressure ratio for impeller and entire stage.

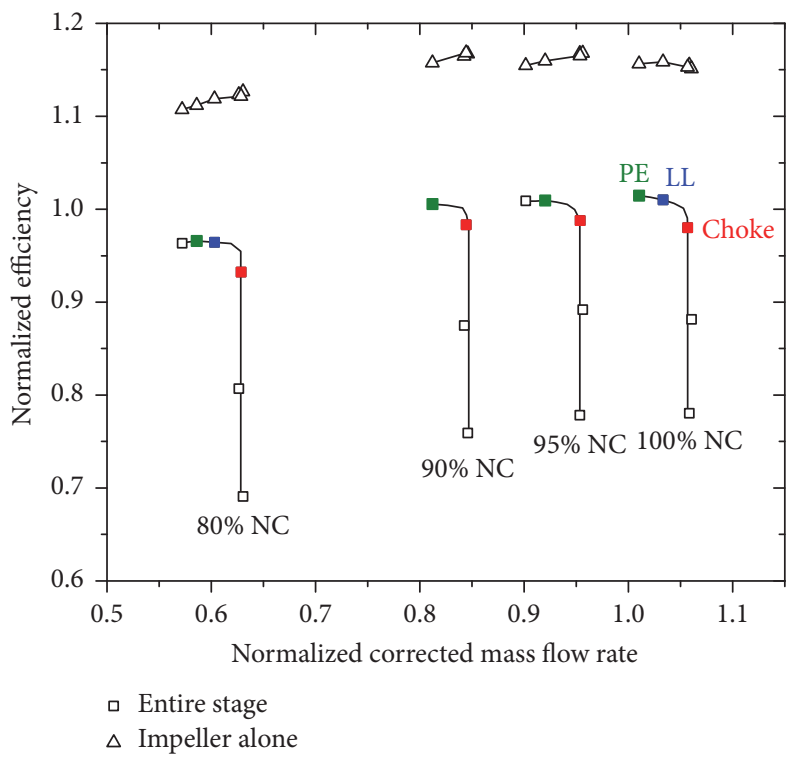

FIGURE 4: Isentropic efficiency for impeller and entire stage.

choke line as loading decreases. In fact, at subsonic inlet conditions from $80 \%$ to $95 \%$ corrected speed, the impeller efficiency increases as loading decreases. At design speed with supersonic inlet conditions, the trend in impeller efficiency lines up with the trend for the entire compressor stage in that they both increase from choke to LL condition.

Figure 5 shows the impeller efficiency versus the inlet tip relative Mach number. The inlet tip relative Mach number is mainly determined by the inlet mass flow rate and prewhirl angle. At supersonic inlet conditions, the impeller efficiency drops with the increase of inlet tip relative Mach number, and there is about a 0.9 -point drop in the efficiency from $95 \%$ to $100 \%$ corrected speed. This trend agrees with the observation from Rodgers [18]. However, at subsonic inlet

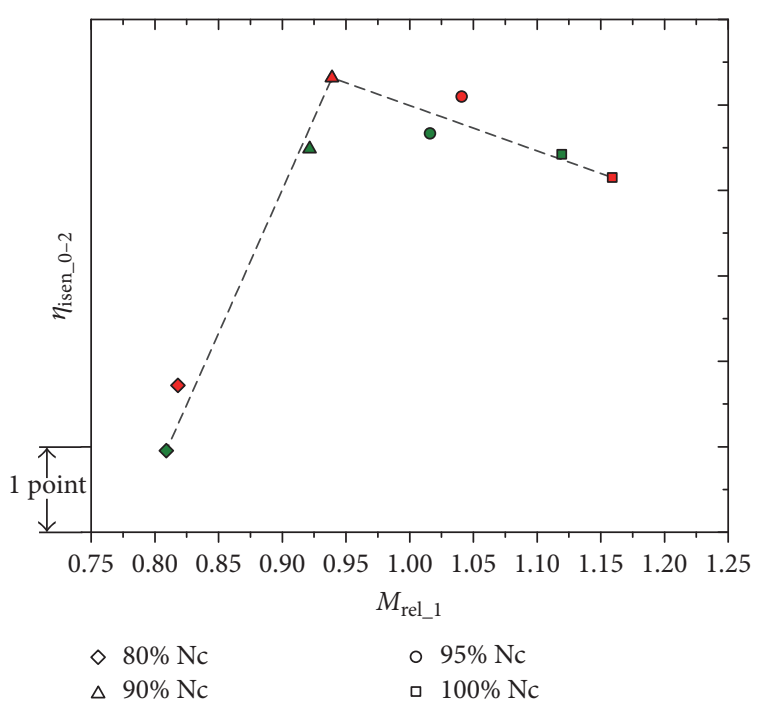

Figure 5: Effect of inlet tip Mach number on impeller efficiency.

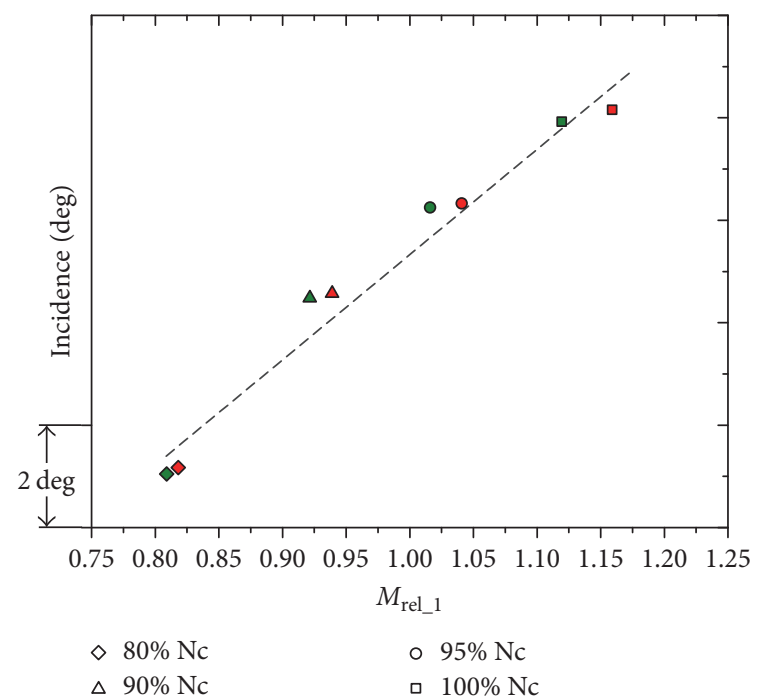

FIGURE 6: Relationship between impeller inlet incidence angle and impeller inlet tip relative Mach number.

conditions, the impeller peak efficiency increases as the inlet tip relative Mach number increases, and there is a 3.5-point improvement in the impeller efficiency from $80 \%$ to $90 \%$ corrected speed.

Figure 6 shows the relationship between inlet tip relative Mach number and impeller inlet incidence angle. The results show a fairly linear correlation between the tip relative Mach number and incidence at the impeller inlet from $80 \%$ to $100 \%$ corrected speed. The incidence angle increases with the increased tip relative Mach number, and there is about a 7.5degree change in the impeller incidence angle from $80 \%$ to $100 \%$ corrected speed.

Furthermore, the detailed distribution of relative Mach number was evaluated from both the steady and timeresolved static pressure measurements along the impeller shroud at each operating condition. Figure 7 shows the 

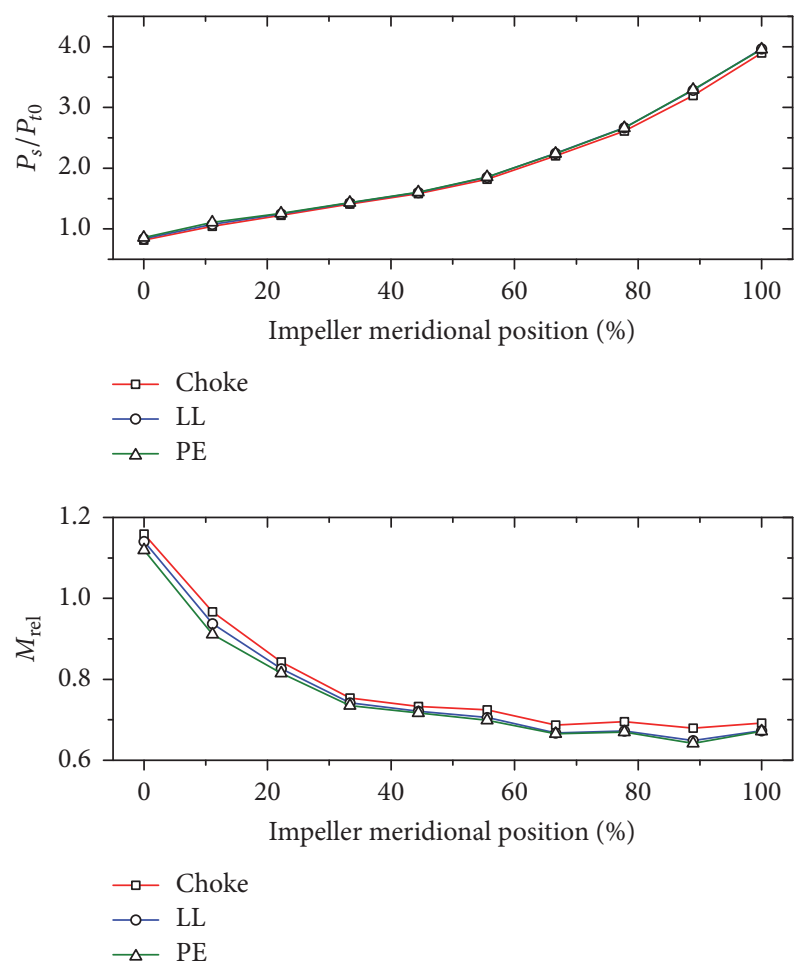

FIgURE 7: Distribution of static pressure and isentropic relative Mach number along impeller shroud at $100 \% \mathrm{~N}_{\mathrm{c}}$.

distribution of static pressure and isentropic relative Mach number along the impeller shroud from LE to TE at $100 \%$ corrected speed. The static pressure is normalized by the areaaveraged total pressure measured at station 0 . The isentropic relative Mach number along the impeller shroud is derived from the equation for conservation of rothalpy and the isentropic flow assumption $[3,8]$. It is calculated as follows:

$$
M_{\mathrm{rel}}=\frac{\sqrt{2\left(I_{1}-h_{s}+U^{2} / 2\right)}}{a},
$$

where $I_{1}$ represents rothalpy at impeller inlet, $h_{s}$ is static enthalpy, $U$ is tangential velocity, and $a$ is speed of sound. Results at three different loading conditions from choke to peak efficiency (as indicated in Figures 3 and 4) are presented. The inlet tip relative Mach number is about 1.15. The inlet tip relative Mach number is obtained from static pressure at the impeller LE and the total pressure and total temperature measured at station 0 , assuming isentropic flow through the inlet housing.

At $100 \%$ corrected speed, the majority of static pressure rise (nearly $81 \%$ ) is achieved in the knee and exducer because of the centrifugal effect, with only about $19 \%$ of the static pressure rise achieved in the inducer. In contrast, over $86 \%$ of relative diffusion occurs in the inducer. Since all the losses occur in the relative diffusion process, the inducer strongly influences impeller efficiency. Additionally, the variation in the shape of the static pressure distribution from choke to peak efficiency is small. In contrast, the distributions of isentropic relative Mach number deviate starting at the
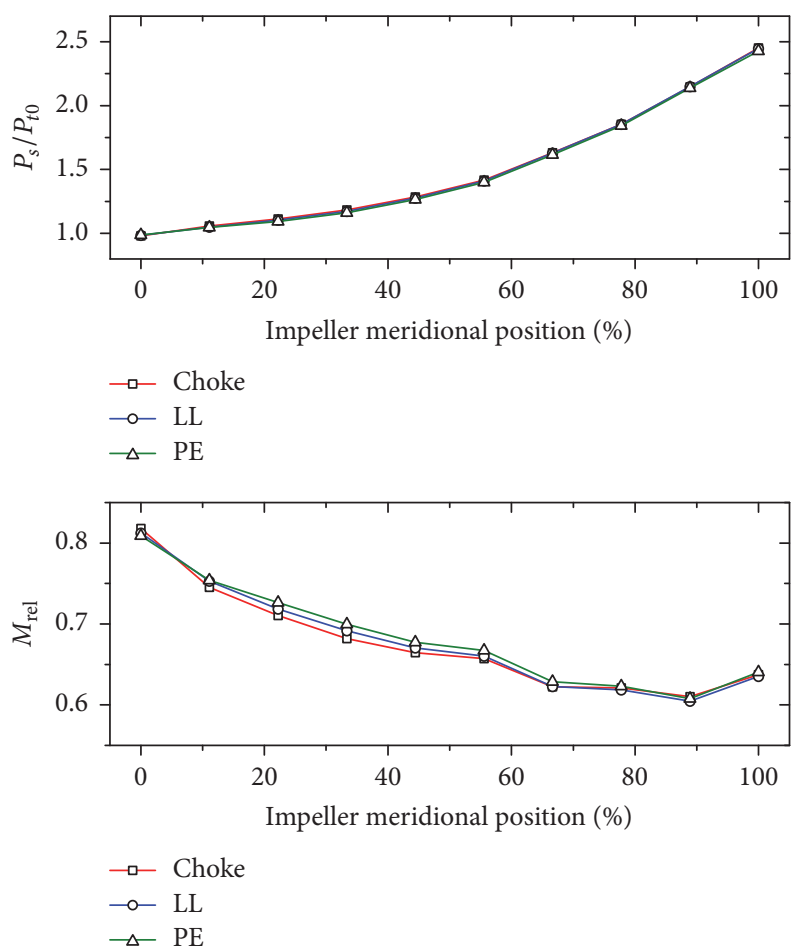

FIGURE 8: Distribution of static pressure and isentropic relative Mach number along impeller shroud at $80 \% \mathrm{~N}_{\mathrm{c}}$.

impeller leading edge. The relative Mach number distribution at $\mathrm{LL}$ and $\mathrm{PE}$ conditions matches those at the knee and exducer, but they are different at the inducer, which leads to the differences in impeller efficiency.

Distributions of static pressure and isentropic relative Mach number along the impeller shroud at subsonic inlet conditions are shown in Figure 8 using the data at $80 \%$ corrected speed. The inlet tip relative Mach number is around 0.82 , and results at three different loading conditions from choke to $\mathrm{PE}$ are presented. The normalized static pressure distributions are similar from choke to peak efficiency. At $80 \%$ corrected speed, about $12 \%$ of the static pressure rise is achieved in the inducer, which is 7 points lower compared to that at $100 \%$ corrected speed. This indicates more loss in the impeller inducer at $80 \%$ corrected speed. Approximately $65 \%$ of the relative diffusion occurs in the inducer, which is 21 points less than the value at $100 \%$ corrected speed. At both $80 \%$ and $100 \%$ corrected speeds, the majority of diffusion is achieved in the inducer while the majority of the static pressure rise occurs in the knee and exducer. This once again highlights the merit of the relative diffusion effectiveness as an indicator of design quality since it removes the bias of using static pressure rise, which is mostly achieved via centrifugal effects.

Figure 9 shows the distribution of static pressure and isentropic relative Mach number at $\mathrm{PE}$ conditions from $80 \%$ corrected speed to $100 \%$ corrected speed. The inlet tip relative Mach number covers both subsonic and supersonic flow conditions. The relative Mach numbers are normalized by the values at the leading edge. The normalized static pressure 

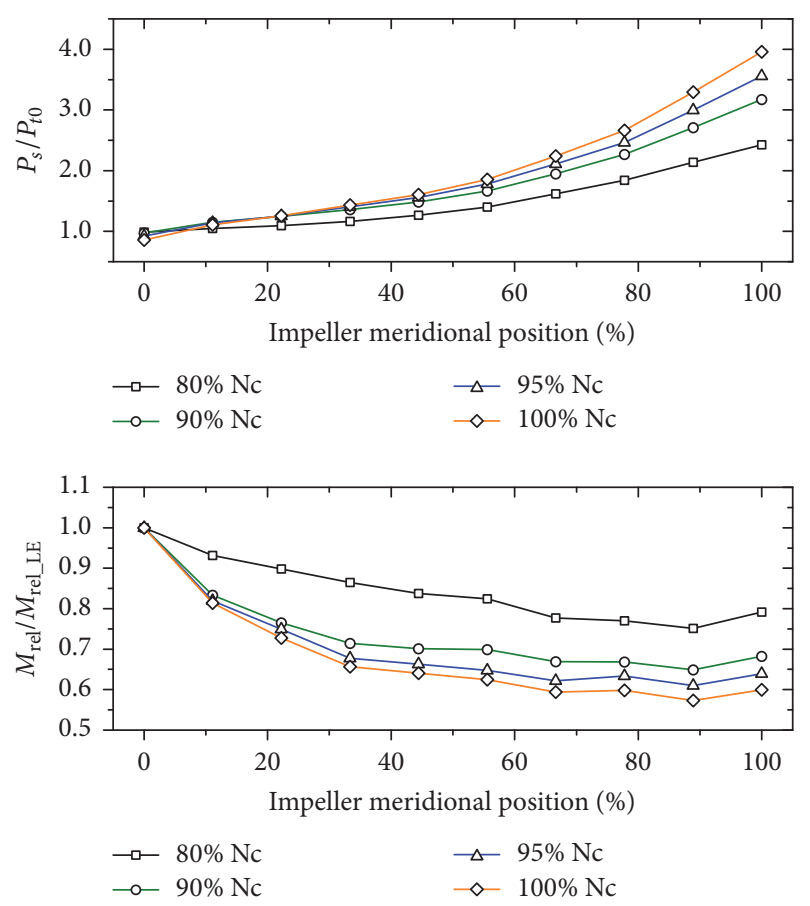

FIGURE 9: Distribution of static pressure and isentropic relative Mach number along impeller shroud at PE conditions.

distributions from $90 \%$ to $100 \%$ corrected speed agree well in the inducer, but they deviate starting at the beginning of the radial turn. However, differences in static pressure distributions start at the LE for $80 \%$ corrected speed, with $69 \%$ less static pressure rise in the inducer. Thus, there is more loss generated in the inducer at $80 \%$ corrected speed and, therefore, lower impeller efficiency (by 3.6 points as shown in Figure 5). The same conclusion is drawn from the isentropic relative Mach number distributions from $80 \%$ to $100 \%$ corrected speed. Additionally, the majority of the loss occurs in regions near the inducer throat.

Furthermore, time-resolved static pressure along the blade chord is processed to reveal more flow features in the inducer. The static pressure is ensemble-averaged using data from 500 rotor revolutions. Figure 10 shows the contours of static pressure and relative Mach number on the shroud surface at the peak efficiency condition. The static pressure is normalized by the inlet total pressure measured at compressor inlet, and the relative Mach number is derived from static pressure and compressor inlet conditions assuming isentropic flow.

The presence of the main blade is indicated by the large gradient between the pressure surface (PS) and the suction surface (SS). In contrast, the presence of the splitter blade is noticeable but less obvious, as indicated by the discontinuity in static pressure contour at mid-passage. A deficit in static pressure corresponds to increased isentropic relative Mach number and vice versa. In addition, both contours show very repeatable flow features in each blade passage with negligible variability due to blade-to-blade differences. Therefore, contours for a single blade passage are used in Figures 11-14. The

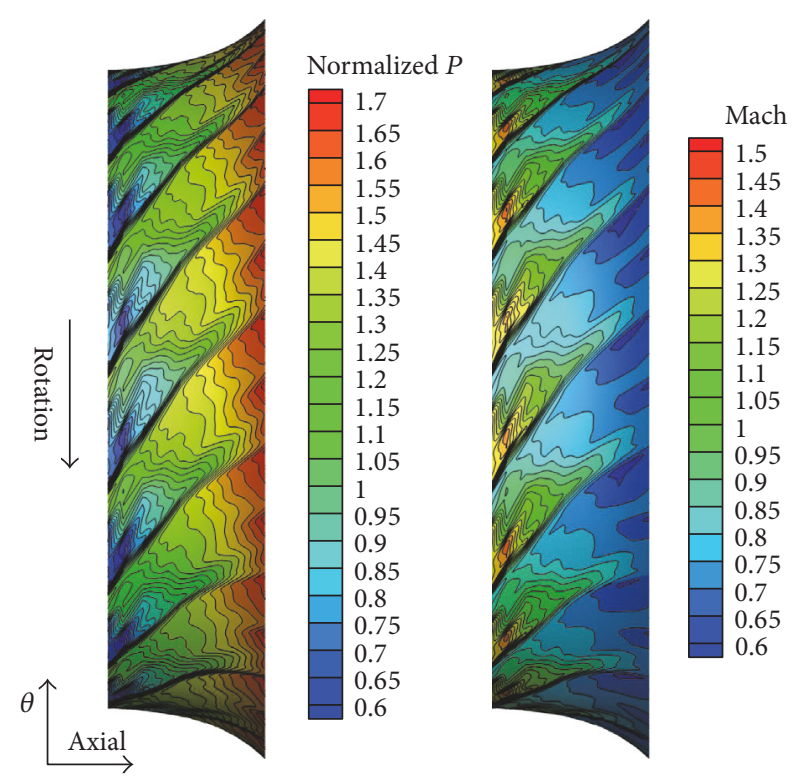

FIgURE 10: Contour of static pressure and isentropic relative Mach number at peak efficiency condition.

contours of a single blade passage were obtained by passageaveraging the ensemble-averaged properties across the entire annulus.

Contours of isentropic relative Mach number derived from time-resolved static pressure measurements at $100 \%$ corrected speed from choke to peak efficiency conditions are shown in Figure 11. The isentropic relative Mach number is calculated using (1). The inlet conditions at 100\% corrected speed are supersonic. There is a significant gradient in Mach number in the pitchwise direction, with high Mach numbers near the SS and low Mach numbers near the PS. Compared to flow on the SS, the flow on the PS gets diffused better, with lower Mach number near the PS, and this applies to both the main and splitter blades. Shock waves near the inducer throat are observed at all three loading conditions, and their positions are shown in the sketch. The position of shock wave is represented by the isolines with isentropic relative Mach number equal to unit. The shock wave forms on the pressure surface near the impeller LE, which further extends into the blade passage. The position of the shock wave changes with loading condition: it moves towards the impeller leading edge from choke to peak efficiency as the inlet tip relative Mach number decreases. Additionally, the corresponding contours of normalized static pressure at $100 \%$ corrected speed are shown in Figure 12. At all three loading conditions, there exists a low-pressure zone in the semiopen region near the suction surface. This is because of the high velocity flow upstream of the shock wave, as shown in the sketch. Both contours get skewed near the entrance of the inducer because of the presence of the shock wave and its associated interactions, with contour lines parallel to the trajectory of the shock waves.

Figure 13 shows contours of isentropic relative Mach number from choke to peak efficiency at $80 \%$ corrected speed 


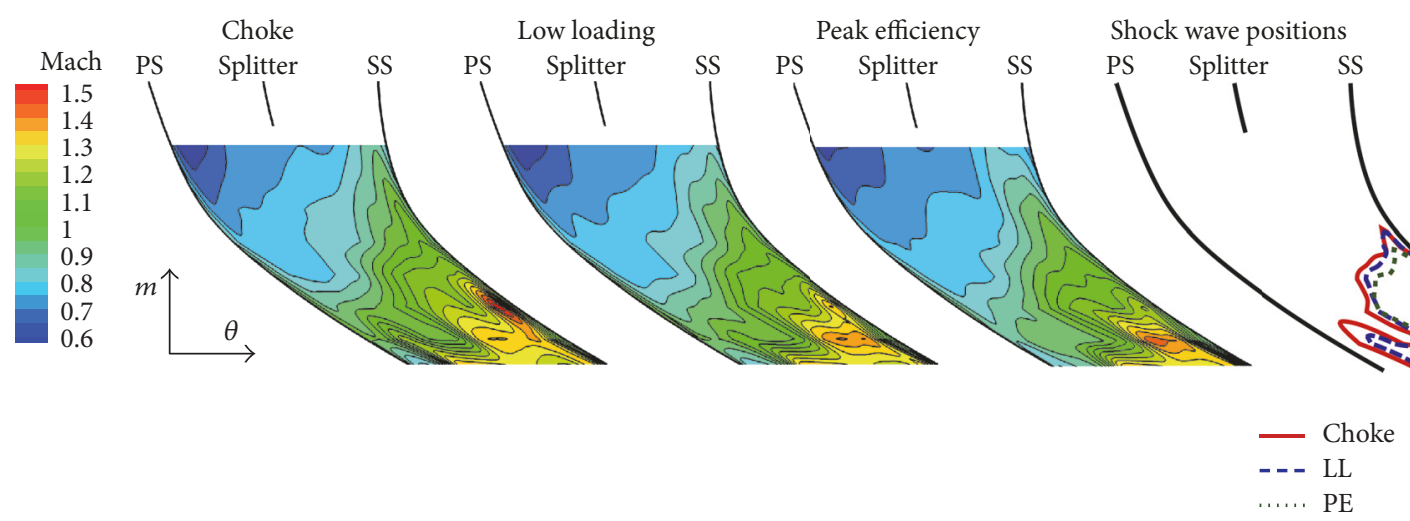

FIGURE 11: Contour of isentropic relative Mach number along the impeller shroud at 100\% Nc.
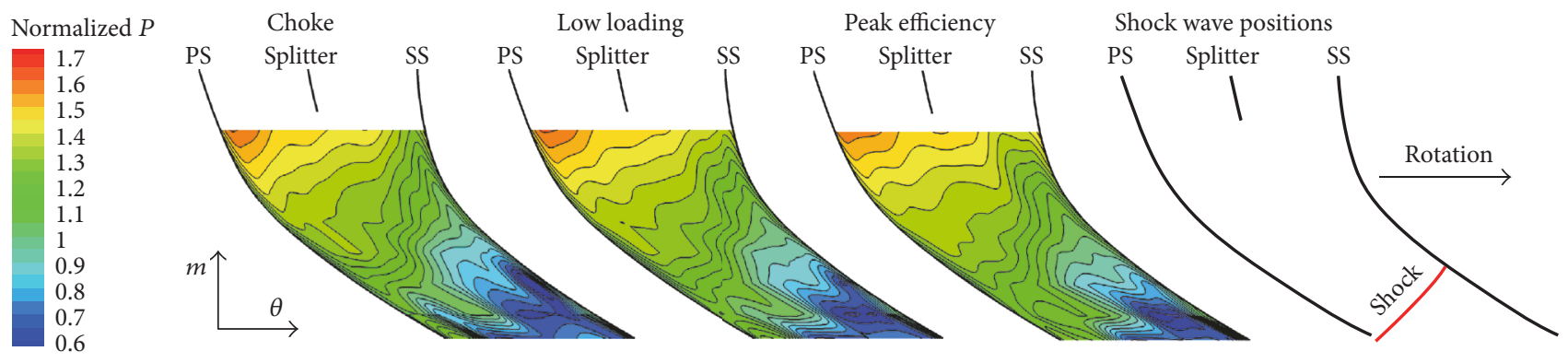

FIGURE 12: Contour of normalized static pressure along the impeller shroud at $100 \% \mathrm{Nc}$.

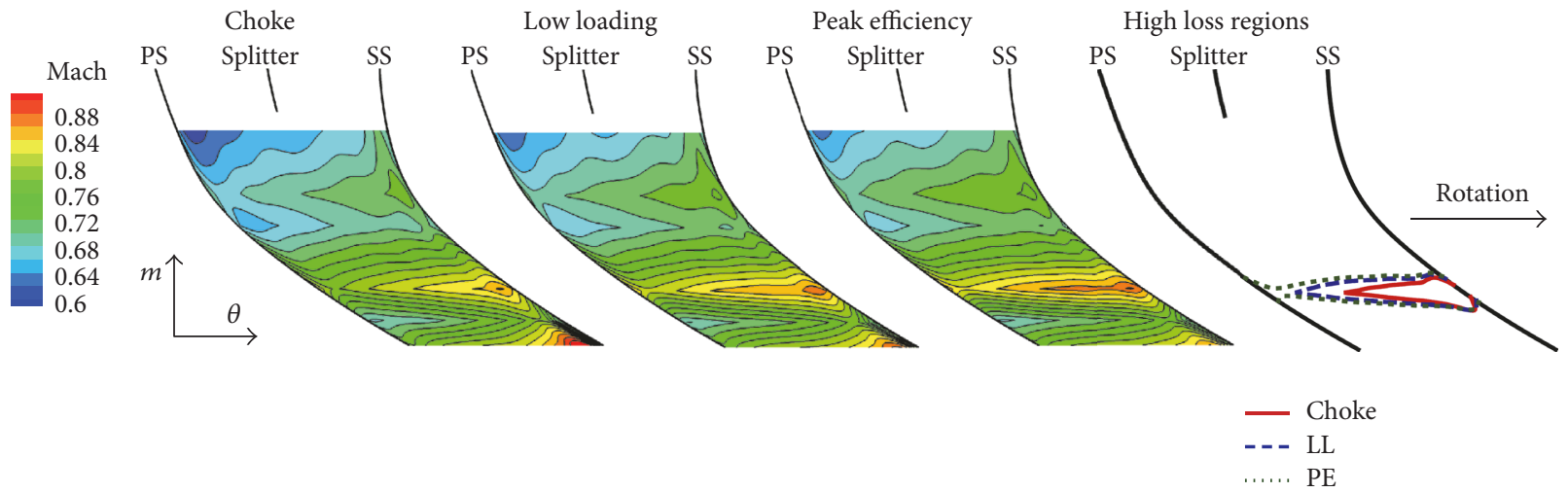

FIGURE 13: Contour of isentropic relative Mach number along the impeller shroud at $80 \% \mathrm{Nc}$.

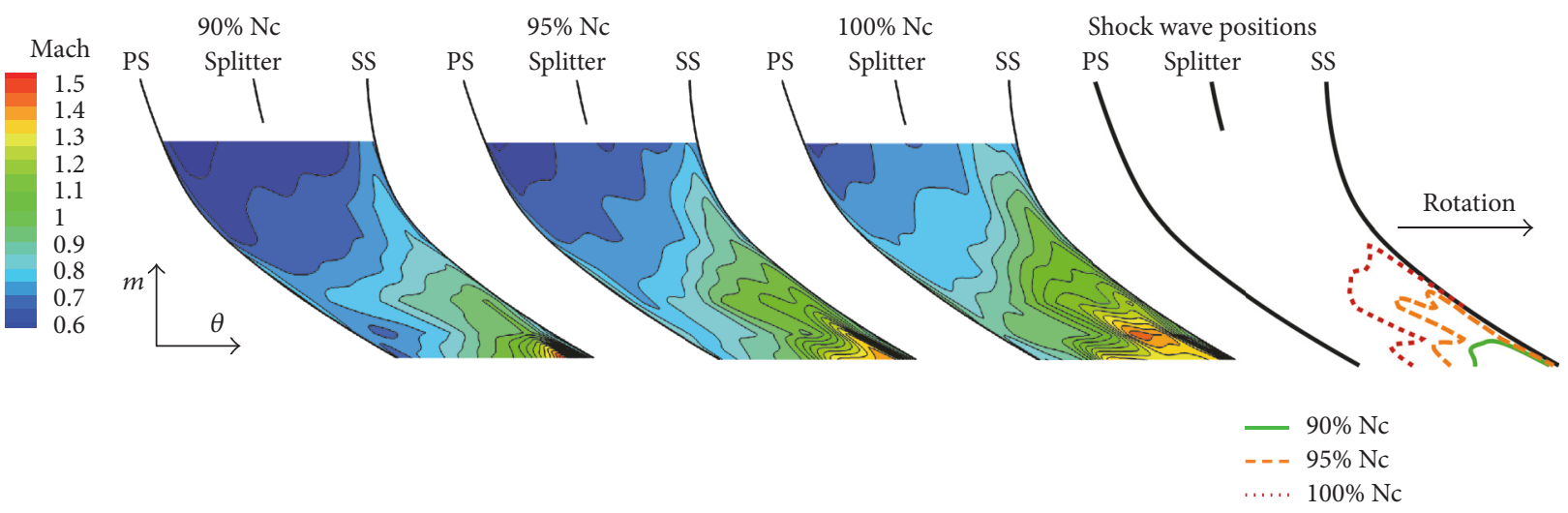

FIGURE 14: Contour of isentropic relative Mach number along the impeller shroud at PE conditions. 
for subsonic inlet conditions. The relative Mach number gradient in the pitchwise direction is much smaller compared to that at $100 \%$ corrected speed. The contour lines are not skewed but are parallel to the impeller leading edge. However, there is a high Mach number zone observed near the inducer throat area at all three loading conditions. The high Mach number represents a deficit in static pressure and additional loss generation. The high-loss region stems from the suction surface and stretches towards the pressure surface. The area of this high-loss region, as shown in the sketch, grows as the loading increases from choke to peak efficiency. It covers $60 \%$ pitch at choke and reaches the pressure surface at peak efficiency. The existence of this high-loss region near the throat in the inducer explains the low efficiency of the impeller at $80 \%$ corrected speed. Additionally, the increased area for the high-loss region from choke to peak efficiency explains the decrease in impeller efficiency as the loading of the entire compressor stage increases. Despite the growth of this high-loss region with increased loading, it is limited to the rear portions of the impeller throat, which agrees with observations from Senoo et al. [2] .

Figure 14 shows the contours of isentropic relative Mach number at peak efficiency conditions from $90 \%$ to $100 \%$ corrected speed. The inlet conditions are supersonic at all three loading conditions, and there are shock waves observed in the inducer, as illustrated in the sketch. The subsonic inlet conditions at $90 \%$ corrected speed calculated from the steady measurements were underpredicted. This is caused by the steady measurements, which do not include the pressure gradient between the SS and PS. There is strong pressure gradient from PS to SS with lower static pressure near impeller SS. This potential field redistributes the flow and enters into impeller eye with flow of higher relative velocity near the SS and lower relative velocity near the PS, which renders a transonic inlet condition at $90 \%$ corrected speed. However, the relative velocity derived from the time-averaged pressure from the steady measurements represents the mean relative velocity, the value of which is between the lower relative velocity on PS and higher relative velocity on the SS. In the present study, it calculates a subsonic inlet condition at $90 \%$ corrected speed. Additionally, the shock wave position in the inducer is closely related to the inlet tip relative Mach number. As the inlet tip Mach number increases, the shock wave moves downstream.

\section{Conclusions}

This paper presents a detailed analysis of transonic impeller aerodynamics using data acquired from both steady and time-resolved static pressure measurements along the impeller shroud. The impeller is a high-speed, high-pressureratio wheel used in aerogas turbine engines. The experiment was conducted on the single stage centrifugal compressor (SSCC) facility in compressor research laboratory at Purdue University. Data were acquired from choke to near-surge at four different corrected speeds $(\mathrm{Nc})$, from $80 \%$ to $100 \%$ design speed, which covers both subsonic and supersonic inlet conditions. By using the parameter isentropic relative Mach number derived from shroud static pressure measurements, the relative diffusion together with shock wave position could be characterized. The information provides a measure of the flow inside the impeller.

Inside the impeller, the isentropic relative Mach numbers along the impeller shroud show that the majority of diffusion is achieved in the inducer at both subsonic and supersonic inlet conditions. The inducer diffuses the flow more efficiently when the inlet tip relative Mach numbers are close to unity at $90 \%$ and $95 \%$ corrected speed, and it becomes less efficient as the inlet tip relative Mach number departs from unity at $80 \%$ and $100 \%$ corrected speed, particularly in the subsonic region.

Shock waves emerging near the LE of the pressure surface were observed from $90 \%$ to $100 \%$ corrected speed with fastresponse pressure measurements. The position of the shock wave changes relative to the inlet tip Mach number. It moves towards the impeller exit as the inlet tip relative Mach number increases. The shock wave at $100 \%$ corrected speed is near the inducer throat, and it moves slightly upstream towards the impeller LE with the increase in loading from choke to peak efficiency. While there is no shock for the subsonic conditions at $80 \%$ corrected speed, there is a high-loss region around the inducer throat. It stems from the suction surface and expands towards the pressure surface as the loading increases from choke to PE.

Static pressure measurements acquired along the impeller shroud are critical in evaluating the impeller performance. However, the relative Mach number calculated from steady static pressure measurements is underestimated because of the pressure gradient from pressure side to suction side in the blade passage. A subsonic value of inlet tip relative Mach number obtained from steady static pressure measurements does not guarantee shock-free flow in the inducer. Compared to the steady static pressure measurements, time-resolved pressure provides more insight for characterizing the impeller flow. They can be used to identify the high-loss regions and shock wave positions.

\section{Nomenclature}
a: Speed of sound
$h$ : Enthalpy
I: Rothalpy
$M$ : Mach number
$P$ : Pressure
$U$ : Blade speed.

\section{Subscripts}

isen: Properties derived from isentropic assumption

rel: Properties in relative frame coordinate

$s: \quad$ Static properties

$t$ : Stagnation properties.

\section{Conflicts of Interest}

The authors declare that there are no conflicts of interest regarding the publication of this paper. 


\section{Acknowledgments}

This research has been sponsored by Honeywell, Inc., and this support is most gratefully acknowledged. The authors also wish to thank Honeywell for granting permission to publish this work. Additionally the guidance and advice offered by Mr. Darrell James and Dr. Rakesh Srivastava of Honeywell were very valuable to this project. Assistance from Herbert Harrison and Amelia Brooks at the Purdue Compressor Research Laboratory during data acquisition was also very much appreciated.

\section{References}

[1] C. Rodgers and L. Sapiro, "Design considerations for highpressure- ratio centrifugal compressors," in Proceedings of the ASME Gas Turbine Conference, 72-GT-91 pages, 1972.

[2] Y. Senoo, H. Hayami, Y. Kinoshita, and H. Yamasaki, "Experimental study on flow in a supersonic centrifugal impeller," Journal of Engineering for Power-Transactions of the ASME, vol. 101, no. 1, p. 32, 1979.

[3] H. Hayami, Y. Senoo, and H. Ueki, "Flow in the inducer of a centrifugal compressor measured with a laser velocimeter," Journal of Engineering for Gas Turbines and Power, vol. 107, no. 2, pp. 534-540, 1985.

[4] H. Krain, B. Hoffmann, and H. Pak, "Aerodynamics of a centrifugal compressor impeller with transonic inlet conditions," in Proceedings of the ASME 1995 International Gas Turbine and Aeroengine Congress and Exposition, GT 1995, USA, June 1995.

[5] H. Krain, G. Karpinski, and M. Beversdorff, "Flow analysis in a transonic centrifugal compressor rotor using 3-component laser velocimetry," in Proceedings of the ASME Turbo Expo 2001: Power for Land, Sea, and Air, GT 2001, USA, June 2001.

[6] S. Ibaraki, H. Higashimori, and T. Matsuo, "Flow investigation of a transonic centrifugal compressor for turbocharger," in Proceedings of the 23nd CIMAC Congress Proceedings, pp. 339346, 2001 .

[7] S. Ibaraki, T. Matsuo, H. Kuma, K. Sumida, and T. Suita, "Aerodynamics of a transonic centrifugal compressor impeller," Journal of Turbomachinery, vol. 125, no. 2, pp. 346-351, 2003.

[8] S. Ibaraki, K. Sumida, and T. Suita, "Design and off-design flow fields of a transonic centrifugal compressor impeller," in Proceedings of the 2009 ASME Turbo Expo, pp. 1375-1384, USA, June 2009.

[9] M. Marconcini, F. Rubechini, A. Arnone, and S. Ibaraki, "Numerical investigation of a transonic centrifugal compressor," Journal of Turbomachinery, vol. 130, no. 1, Article ID 011010, 2008.

[10] M. Marconcini, F. Rubechini, A. Arnone, and S. Ibaraki, "Design and off-design numerical investigation of a transonic double-splitter centrifugal compressor," in Proceedings of the 2008 ASME Turbo Expo, Germany, June 2016.

[11] H. Higashimori, K. Hasagawa, K. Sumida, and T. Suita, "Detailed flow study of mach number 1.6 high transonic flow with a shock wave in a pressure ratio 11 centrifugal compressor impeller," Journal of Turbomachinery, vol. 126, no. 4, pp. 473481, 2004.

[12] N. Buffaz and I. Trébinjac, "Detailed analysis of the flow in the inducer of a transonic centrifugal compressor," Journal of Thermal Science, vol. 21, no. 1, pp. 1-12, 2012.
[13] F. Lou, H. M. Harrison, J. C. Fabian, N. L. Key, D. K. James, and R. Srivastava, "Development of a centrifugal compressor facility for performance and aeromechanics research," in Proceedings of the ASME Turbo Expo 2016: Turbomachinery Technical Conference and Exposition, GT 2016, Republic of Korea, June 2016.

[14] H. Simon, T. Wallmann, and T. Moenk, "Improvements in performance characteristics of single-stage and multistage centrifugal compressors by simultaneous adjustments of inlet guide vanes and diffuser vanes," in Proceedings of the ASME Gas Turbine Conference, vol. 109, 1986.

[15] R. A. Berdanier, N. R. Smith, J. C. Fabian, and N. L. Key, "Humidity effects on experimental compressor performancecorrected conditions for real gases," Journal of Turbomachinery, vol. 137, no. 3, Article ID 031011, 2015.

[16] F. Lou, J. Fabian, and N. L. Key, "The effect of gas models on compressor efficiency including uncertainty," Journal of Engineering for Gas Turbines and Power, vol. 136, no. 1, Article ID 012601, 2014.

[17] E. W. Lemmon, M. L. Huber, and M. O. McLinden, "NIST Standard Reference Database 23: Reference Fluid Thermodynamic and Transport Properties-REFPROP, Version 9.1, National Institute of Standards and Technology, Standard Reference Data Program," Gaithersburg, MD, USA, 2013.

[18] C. Rodgers, "Centrifugal compressor inducer," in Proceedings of the ASME Gas Turbine Conference, 98-GT-032 pages, 1998. 


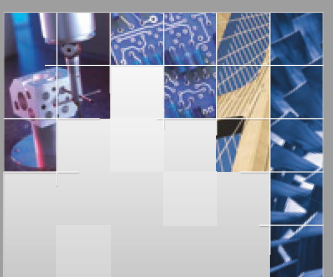

\section{Enfincering}
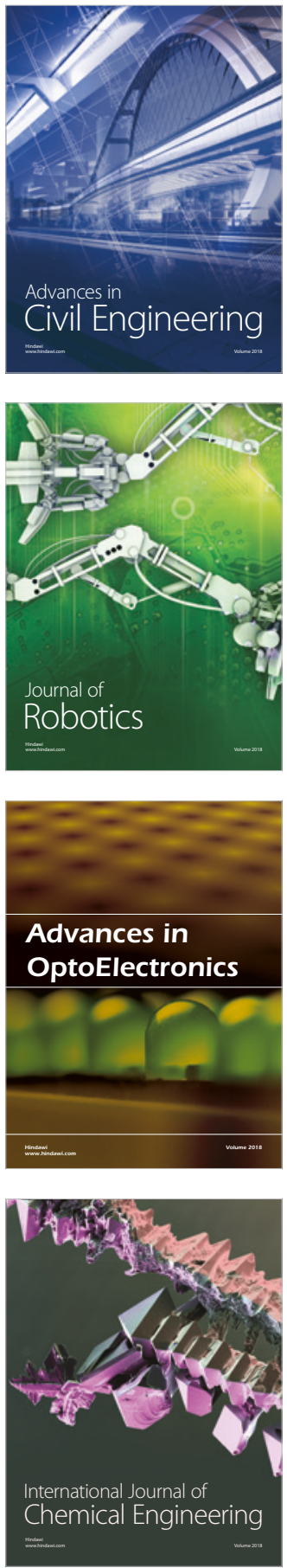

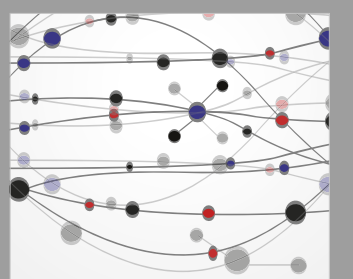

\section{Rotating \\ Machinery}

The Scientific World Journal

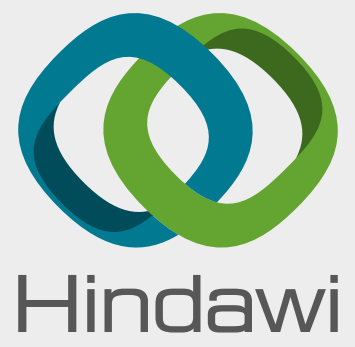

Submit your manuscripts at

www.hindawi.com
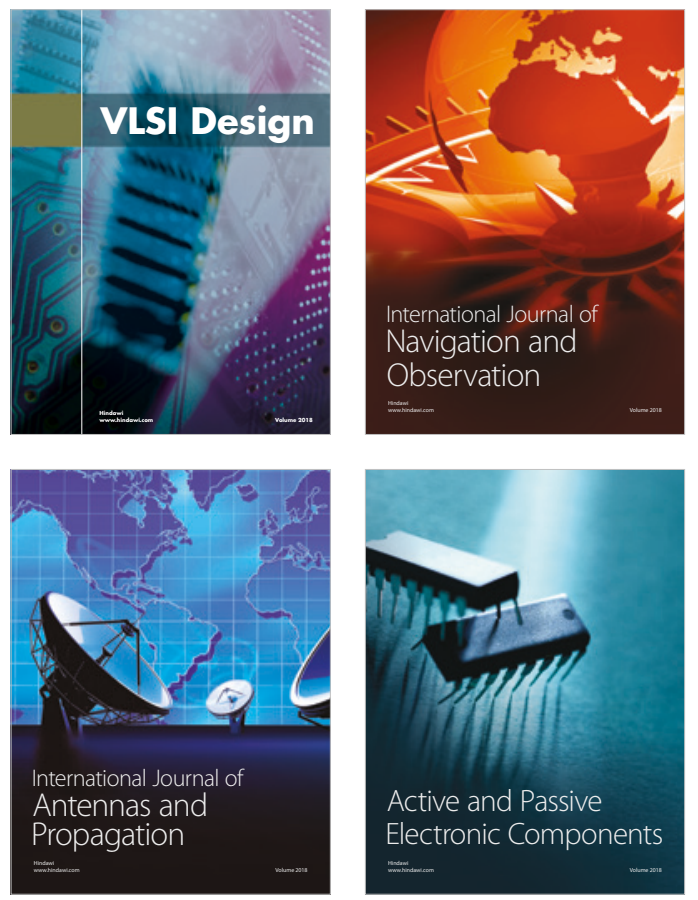
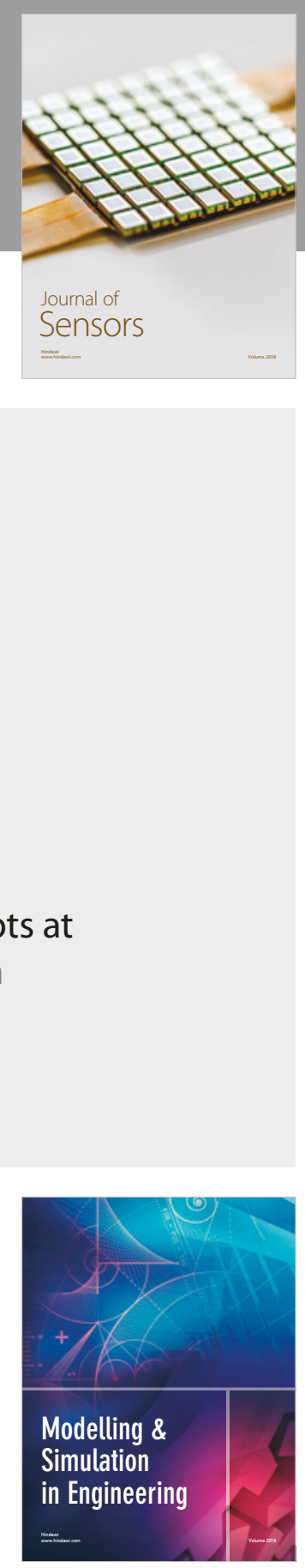

\section{Advances \\ Multimedia}
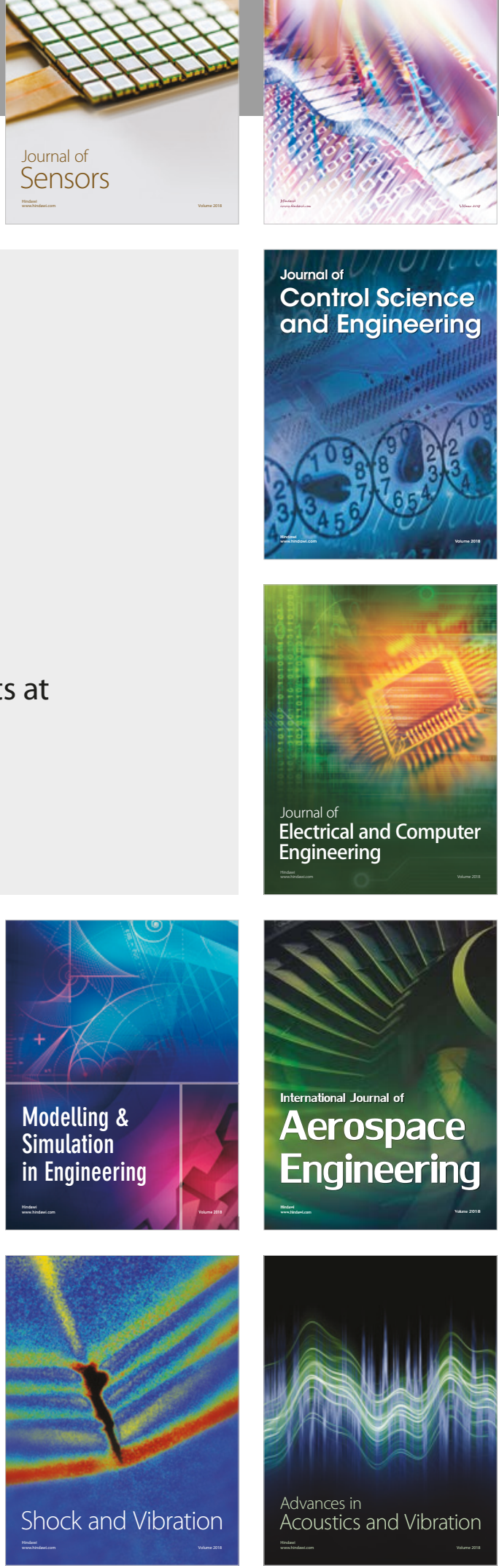